\title{
PERFIL EPIDEMIOLÓGICO DOS ÓBITOS POR CAUSAS ACIDENTAIS EM CRIANÇAS E ADOLESCENTES NO VALE DO JEQUITINHONHA
}

Endi Lanza GALVÃO ${ }^{1}$

Emerson Cotta BODEVAN ${ }^{2}$

Delba Fonseca SANTOS ${ }^{3}$

\begin{abstract}
${ }^{1}$ Mestre em Saúde, Sociedade e Ambiente. Universidade Federal dos Vales do Jequitinhonha e Mucuri (UFVJM).endilanza@yahoo.com.br

${ }^{2}$ Doutor em Estatística. Universidade Federal dos Vales do Jequitinhonha e Mucuri (UFVJM). bodevan.ec@gmail.com

${ }^{3}$ Doutora em Saúde Coletiva. Universidade Federal dos Vales do Jequitinhonha e Mucuri (UFVJM). delbafonseca@yahoo.com.br
\end{abstract}

Recebido em: 22/08/2016 - Aprovado em: 30/06/2017 - Disponibilizado em: 01/07/2017

\begin{abstract}
RESUMO:
Objetivou-se caracterizar o perfil epidemiológico dos óbitos infanto-juvenis por causas acidentais, ocorridos na macrorregião de saúde Jequitinhonha, situada no Vale do Jequitinhonha, e compará-lo com a macrorregião de saúde Centro, do estado de Minas Gerais. Realizou-se análise quantitativa a partir de dados coletados do Sistema de Informações sobre Mortalidade, entre 1998 a 2013. O levantamento englobou dados de crianças e adolescentes com idade de um a dezenove anos, considerando-se os óbitos por causas externas acidentais, identificados na décima revisão da Classificação Internacional de Doenças (CID-10). Os resultados apontam que a macrorregião Centro apresentou maior ocorrência de óbitos por acidentes de transportes $(63,8 \%)$ quando comparado ao Vale do Jequitinhonha (52,5\%), que por sua vez prevaleceu com óbitos por outras causas de traumatismos $(47,5 \%)(\mathrm{p}=0,003)$. O levantamento desta problemática pode orientar abordagens técnico-científicas para minimização da ocorrência de acidentes com crianças e adolescentes.
\end{abstract}

Palavras-Chave: Prevenção de Acidentes. Epidemiologia. Mortalidade. Criança. Saúde Pública.

\section{ABSTRACT:}

This study aimed to characterize the epidemiological profile of children and youth deaths from accidental injuries occurring in the Jequitinhonha region, located in the Jequitinhonha Valley, and compare it with the Center of Minas Gerais state. We conducted quantitative analysis from data collected from the Mortality Information System from 1998 to 2013. The survey encompassed data from children and adolescents aged from one to nineteen, considering the accidental deaths by external causes, identified according to the tenth revision of the International Classification of Diseases. The results show that region center showed higher incidence of deaths due to transport accidents $(63.8 \%)$ compared to the Jequitinhonha Valley (52.5\%), which in turn prevailed with deaths from other causes of trauma (47.5 $\%)(p=0.003)$. The survey of this problem can guide technical and scientific approaches to minimize the occurrence of accidents in the age group studied.

Keywords: Accident Prevention. Epidemiology. Mortality. Child. Public Health.

\section{Introdução}

A atual situação de saúde brasileira está marcada por uma transição demográfica acelerada e por uma situação de tripla carga de doenças em um contexto onde as causas externas ocupam um importante patamar. A décima revisão da Classificação Internacional de Doenças (CID-10) usa a denominação de acidente ou lesões não intencionais para alguns tipos de causas externas. No entanto, a 
literatura internacional mais recente tem evitado essa denominação uma vez que essa palavra é comumente ligada ao conceito de “evento fortuito, não previsível” e, portanto, não passível de prevenção (GAWRYSZEWSKI; KOIZUMI; MELLOJORGE, 2004). A análise da carga de doença no Brasil mostra que as causas externas representam $10,2 \%$ da carga global de doenças no país, medidas em anos de vida perdidos ajustados por incapacidade (AVAIs) (SCHRAMM et al., 2004).

Lesões não intencionais em crianças representam um importante problema de saúde pública (ORGANIZAÇÃO MUNDIAL DE SAÚDE, 2008; BRASIL, 2012) e até recentemente pouca atenção tem sido dada a esta demanda em países de média e baixa renda (ORGANIZAÇÃO MUNDIAL DE SAÚDE, 2008). A literatura aponta que $98 \%$ das mortes de crianças e jovens por causas externas ocorrem nos países em desenvolvimento, apontando como fatores de risco a pobreza, mãe solteira e jovem, baixo nível de educação materna, habitações pobres, famílias numerosas e uso de álcool e drogas pelos pais (BLANK, 2002). Nesse sentido, os acidentes guardam relação com uma gama de fatores, como as condições ambientais, físicas, culturais e sociais da família, o estilo de vida dos pais e suas condições de vida e trabalho, urbanização, marginalidade, desemprego, desigualdade social, superpopulação, miséria, educação, estresse, condições impróprias de moradia, vigilância insuficiente, entre outros (MARTINS, 2006).

Desta forma, a heterogeneidade regional do território brasileiro persistente ao longo de décadas suscita um perfil diferenciado das causas acidentais em crianças e adolescentes. No Brasil, no ano 2013, ocorreram 20.903 mortes por causas externas em indivíduos com idade de um a dezenove anos. Destas, 9,5\% ocorreram na região centro-oeste e $31,3 \%$ ocorreram na região sudeste do país (BRASIL, 2016). A literatura aponta que, de um modo geral, lactentes e pré-escolares sofrem mais queimaduras, afogamentos, quedas e intoxicações; escolares sofrem mais atropelamentos e quedas de bicicletas; adolescentes estão mais sujeitos a afogamentos, traumas no trânsito e por armas de fogo (BLANK, 2002). No entanto, não se sabe como esta distribuição ocorre entre distintas regiões de um mesmo estado.

Com o objetivo de obter melhor compreensão sobre as causas externas por acidentes em crianças e adolescentes e suas relações com o contexto sócio-cultural, este artigo se propõe a caracterizar o perfil epidemiológico dos óbitos infanto-juvenis por injúrias acidentais, ocorridos na macrorregião de saúde Jequitinhonha, situada no Vale do Jequitinhonha, e compará-lo com a macrorregião de saúde Centro, do estado de Minas Gerais. Espera-se criar evidências que subsidiem pesquisas de campo futuras para 
auxiliar na elaboração de políticas públicas e enfrentamento das lacunas do sistema de saúde.

\section{Metodologia}

Trata-se de um estudo transversal que avaliou os óbitos infanto-juvenis por causas acidentais ocorridas na macrorregião Jequitinhonha, comparadas às ocorridas na macrorregião Centro do estado de Minas Gerais.

As regiões analisadas (Jequitinhonha e Centro) foram definidas conforme divisão das macrorregiões de saúde da Secretaria Estadual de Saúde de Minas Gerais, devidamente discriminadas no Plano Diretor de Regionalização (PDR) da saúde do estado (MINAS GERAIS, 2011).

A macrorregião Jequitinhonha, recentemente renomeada Região Ampliada de Saúde Jequitinhonha, é composta por 29 municípios situados no Vale do Jequitinhonha, apresentando Diamantina como cidade de referência para aproximadamente 296.344 indivíduos (MINAS GERAIS, 2011). Representa uma das treze regiões ampliadas de saúde estabelecidas pelo PDR/MG e apresenta comportamento desigual entre as demais regiões devido ao ciclo de pobreza e às dificuldades de acesso à saúde, vigentes nesta região. O Vale do Jequitinhonha, situado no nordeste do estado e banhado pelo rio Jequitinhonha, é uma região que se caracteriza pelos maiores índices de vulnerabilidade social no estado de Minas Gerais, com elevados índices de desnutrição, mortalidade e analfabetismo (INSTITUTO DE PESQUISA ECONÔMICA APLICADA, 2015). Segundo Censo de 2010, esta região apresentou $79,42 \%$ das crianças em situação domiciliar de baixa renda (com renda menor do que meio salário mínimo) (BRASIL, 2016).

Com 48,5\% das crianças em situação de baixa renda (Censo 2010) (BRASIL, 2016), a região ampliada de saúde Centro possui como referência o município de Belo Horizonte, capital do estado de Minas Gerais, é referência para 104 municípios e abrange uma população de 6.357 .604 habitantes.

Para caracterizar o perfil dos óbitos por causas acidentais, foram extraídos dados do Sistema de Informação sobre Mortalidade (SIM), publicados pelo Departamento de Informática do Sistema Único de Saúde (DATASUS), disponíveis no endereço eletrônico do Ministério da Saúde (BRASIL, 2016). O sistema foi acessado no período de abril a maio de 2016.

Dentre as causas externas, foram consideradas apenas as causas relacionadas aos acidentes do Grupo CID 10 (V01-X59). Assim, foram excluídas as causas externas por lesões auto-provocadas intencionalmente (X60-X84), agressões (X85-Y09), eventos com intenção indeterminada (Y10-Y34), intervenções legais e operações de guerra 
(Y35-Y36), complicações de assistência médica e cirúrgica (Y40-Y84), seqüelas por causas externas de morbidade e mortalidade (Y85-Y89) e fatores suplementares (Y90Y98).

A análise temporal foi de 1998 a 2013, último ano em que os dados estavam disponíveis no DATASUS. O levantamento englobou dados de crianças e adolescentes com idade de um a dezenove anos.

Os dados foram analisados utilizandose o programa SPSS versão 22.0. Foram obtidas as freqüências absolutas e percentuais (técnicas de estatística descritiva) e utilizado o teste Qui-quadrado de Pearson e Teste Exato de Fisher. O nível de significância utilizado nas decisões dos testes estatísticos foi de $5 \%$ $(\mathrm{p}<0,05)$.

Figura 1 - Distribuição dos óbitos por causas acidentais nas macrorregiões (a) Centro e (b) Jequitinhonha, para a faixa etária de 1 a 19 anos, no período de 1998 a 2013.

(a)

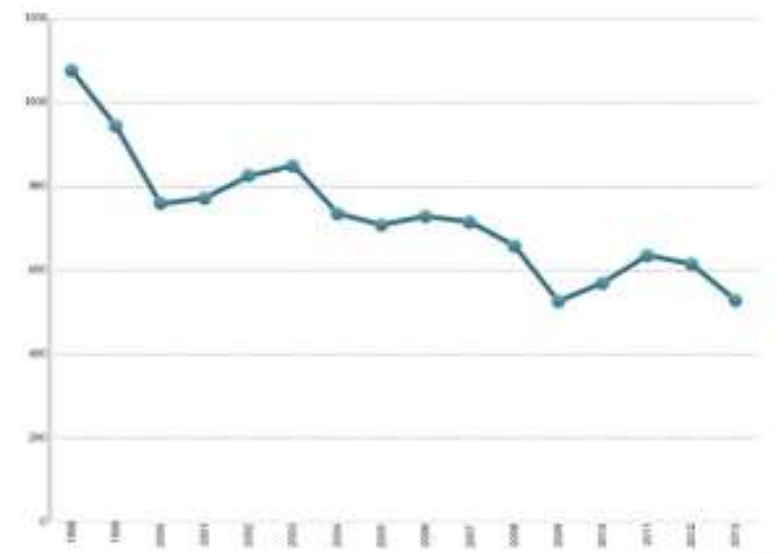

\section{Resultados e Discussão}

No período analisado, o estado de MG apresentou um coeficiente médio de mortalidade por injúrias acidentais infantojuvenis igual a 2,1 casos/1000 habitantes. A macrorregião Jequitinhonha apresentou média de 1,5 casos/1000 habitantes e a região central do estado de 1,9 casos/1000 habitantes. A partir da construção da série histórica para o período de 1998 a 2013, observou-se tendência decrescente na ocorrência de óbitos por acidentes com crianças e adolescentes na macrorregião Centro (Figura 1a), enquanto na macrorregião Jequitinhonha esta apresentação foi oscilante, com tendências crescentes (Figura 1b).

Fonte: MS/SVS/CGIAE - Sistemas de Informações sobre Mortalidade - SIM, Departamento de Informática do Sistema único de Saúde, Ministério da Saúde, Brasil.

Para pensar em formas de prevenir os óbitos por causas acidentais é necessário (b)

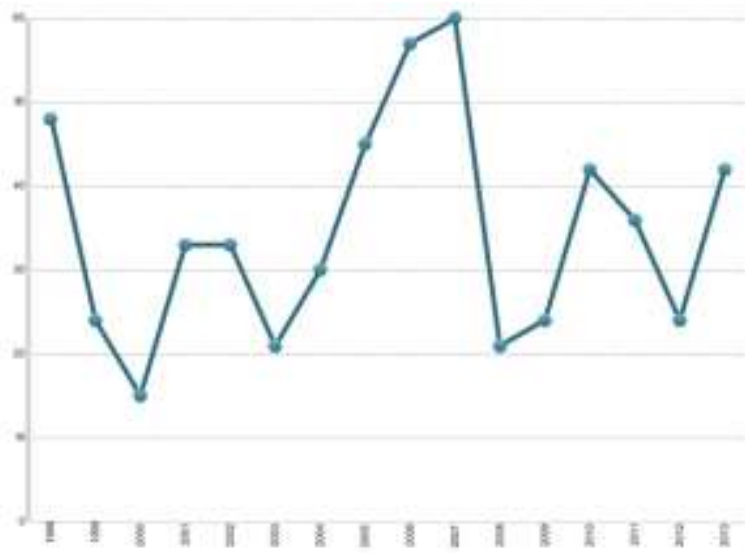

avaliar principalmente os motivos que provocaram estes acidentes. A Organização 
Mundial de Saúde destaca que as lesões não intencionais mais incidentes em crianças e adolescentes são acidentes de trânsito, afogamentos, intoxicações, queimaduras e quedas (ORGANIZAÇÃO MUNDIAL DE SAÚDE, 2008). No presente estudo, ao organizar as causas acidentais infanto-juvenis em dois grandes grupos (acidentes de transportes e acidentes por outras causas externas de traumatismos), proporcionalmente, houve menos óbitos por acidente de transporte na macrorregião Jequitinhonha $(52,5 \%)$ do que na macrorregião Centro (63,8\%). Por outro lado, o Vale do Jequitinhonha apresentou maior ocorrência de acidentes por outras causas externas de traumatismos $(47,5 \%)$ quando comparado com a região central do estado $(36,2 \%)(\mathrm{p}=0,003)$.

A distribuição dos óbitos de acordo com as causas relacionadas aos acidentes do Grupo CID 10 (V01-X59) está apresentada na tabela 1 .

O afogamento e submersão acidentais representaram a maior causa de óbito por acidentes na macrorregião Jequitinhonha $(29,9 \%)$ além de ter sido mais prevalente do que na macrorregião Centro $(19,7 \%)$ $(\mathrm{p}=0,0013)$. O risco de afogamento de crianças menores de 1 ano de idade está associado a piscinas, banheiras e piscinas pequenas portáteis e em consequiência de descuido dos responsáveis; já para as crianças de 1 a 3 anos, as piscinas residenciais representam grande risco de afogamento (PAES; GASPAR, 2005). Entretanto, resposta para elevada prevalência de afogamento e submersão, especificamente no contexto regional do Vale do Jequitinhonha, pode ter relação com práticas de utilização do rio Jequitinhonha e afluentes para banho e pesca.

A mortalidade por contato com animais e plantas venenosas e por exposição às forças da natureza também foram mais prevalentes na macrorregião Jequitinhonha $(5,1$ e $2,8 \%$ respectivamente) do que na macrorregião Centro $(0,8 \%$ para ambas as causas $) \quad(p<0,0001$ e $\quad p=0,0204$, respectivamente). Estes resultados corroboram com o perfil da região estudada. A existência majoritária de pequenas cidades no Vale do Jequitinhonha (GALVÃO; BODEVAN; SANTOS, 2015), que ainda sofrem grandes influências do mundo rural, pode justificar o perfil das causas de acidentes infanto-juvenis encontrados neste estudo.

A macrorregião Jequitinhonha apresentou maior proporção de óbitos de motociclistas envolvidos em acidentes de transportes $(9,6 \%)$ do que a região central do estado (7,7\%). Apesar desta diferença não ter sido estatisticamente significativa, é um resultado que merece atenção. Apesar do índice de motorização crescente na capital e cidades metropolitanas determinarem maior exposição ao risco de acidentes de transportes nestas localidades, cortadas por vias expressas e muito movimentadas, este risco pode estar 
presente no interior de cidades em crescimento(MINAYO, 2009).

Tabela1- Óbitos por ocorrência, de 1 a 19 anos, apresentados por Macrorregião de Saúde segundo Grupo CID10, período de 1998- 2013.

\begin{tabular}{|c|c|c|c|c|}
\hline Causa dos Acidentes & $\begin{array}{c}\text { Macrorregião } \\
\text { Jequitinhonha } \\
\mathbf{N}(\%) \\
\end{array}$ & $\begin{array}{c}\text { Macrorregião } \\
\text { Centro } \\
\mathrm{N}(\%) \\
\end{array}$ & $\mathbf{n}$ & $p$ \\
\hline $\begin{array}{l}\text { Pedestre traumatizado em um acidente de } \\
\text { transporte }\end{array}$ & $19(10,7)$ & $758(19,0)$ & 777 & $0,0089 *$ \\
\hline $\begin{array}{l}\text { Ciclista traumatizado em um acidente de } \\
\text { transporte }\end{array}$ & $2(1,1)$ & $136(3,4)$ & 138 & $0,1534^{*}$ \\
\hline $\begin{array}{l}\text { Motociclista ou ocupante de triciclo traumatizado } \\
\text { em um acidente de transporte }\end{array}$ & $17(9,6)$ & $311(7,7)$ & 328 & $0,4423 *$ \\
\hline $\begin{array}{l}\text { Ocupante automóvel traumatizado em acidente } \\
\text { transporte }\end{array}$ & $16(9,0)$ & $467(11,6)$ & 483 & $0,3545^{*}$ \\
\hline Acidentes de transporte por água & 0 & $3(0,1)$ & 3 & $1 * *$ \\
\hline Outros acidentes de transporte não especificados & $39(22,0)$ & $894(22,2)$ & 933 & $1 *$ \\
\hline Quedas & $1(0,6)$ & $160(4,0)$ & 161 & $0,0346^{*}$ \\
\hline Exposição a forças mecânicas inanimadas & $2(1,1)$ & $88(2,2)$ & 90 & $0,5900 * *$ \\
\hline Exposição a forças mecânicas animadas & 0 & $8(0,2)$ & 8 & $1 * *$ \\
\hline Afogamento e submersão acidentais & $53(29,9)$ & $794(19,7)$ & 847 & $0,0013 *$ \\
\hline Outros riscos acidentais à respiração & $4(2,3)$ & $108(2,7)$ & 112 & $1 * *$ \\
\hline $\begin{array}{l}\text { Exposição à corrente elétrica, à radiação e à } \\
\text { temperaturas e pressões extremas do ambiente }\end{array}$ & $1(0,6)$ & $41(1,0)$ & 42 & $1 * *$ \\
\hline Exposição à fumaça, ao fogo e às chamas & $1(0,6)$ & $106(2,6)$ & 107 & $0,1350 * *$ \\
\hline $\begin{array}{l}\text { Contato com fonte de calor ou substâncias } \\
\text { quentes }\end{array}$ & 0 & $13(0,3)$ & 13 & $1 * *$ \\
\hline Contato com animais e plantas venenosos & $9(5,1)$ & $30(0,8)$ & 39 & $<0,0001 * *$ \\
\hline Exposição às forças da natureza & $5(2,8)$ & $33(0,8)$ & 38 & $0,0204 * *$ \\
\hline $\begin{array}{l}\text { Envenenamento acidental e exposição à } \\
\text { substâncias nocivas }\end{array}$ & $1(0,6)$ & $6(0,2)$ & 7 & $0,2603 * *$ \\
\hline $\begin{array}{l}\text { Exposição acidental a outros fatores não } \\
\text { especificados }\end{array}$ & $7(4,0)$ & $69(1,7)$ & 76 & $0,0396 * *$ \\
\hline Total & 177 & 4025 & 1540 & \\
\hline
\end{tabular}

Em muitas cidades de pequeno e médio porte, prevalentes no Vale do Jequitinhonha, o uso do capacete e da carteira de habilitação não é respeitado e a segurança pública nestas pequenas cidades costuma ser insuficiente para realizar a fiscalização adequada. Neste sentido, adolescentes menores de dezoito anos, e portanto, sem habilitação para dirigir, tornam-se condutores deste tipo de veículo sem a devida formação, o que pode predispor à ocorrência de acidentes, e óbitos por consequência . Além disso, existe evidência de que as menores taxas de utilização de capacete ocorrem entre crianças, adolescentes e idosos, ressaltando que o uso do capacete representa a medida mais viável de proteção individual na ocorrência de acidentes motociclísticos (LIBERATTI et al, 2003).

Em ambas regiões, maior mortalidade por acidentes ocorreu na faixa etária de 15 a 19 anos. Inexperiência, busca de emoções, prazer em experimentar sensações de risco, impulsividade e abuso de álcool ou drogas são 
termos associados aos comportamentos de adolescentes que podem explicar maior acometimento de acidentes nesta fase da vida (BASTOS, ANDRADE, SOARES, 2005). Na macrorregião Jequitinhonha, 46,3\% dos óbitos por acidentes ocorreram nesta faixa etária e na macrorregião Centro, 49,9\%. Analisando ainda este extrato etário, observou-se que na região central do estado e no Vale do Jequitinhonha, a maioria dos óbitos foi relacionada a acidentes de transportes (71,7 e 75,3\%, respectivamente). Assim, políticas públicas direcionadas aos adolescentes, priorizando o desenvolvimento de postura de auto-responsabilidade poderiam ser úteis na prevenção de acidentes.

$\mathrm{Na}$ macrorregião Jequitinhonha, para as faixas etárias de 5 a 9 anos e de 10 a 14 anos, observou-se maior proporção de acidentes relacionados a afogamento, exposição acidental a corrente elétrica, a radiação, a forças da natureza, além de contato com animais e plantas venenosas $(67,2 \%)$. Na macrorregião Centro, óbitos por estes fatores, nestas faixas etárias, aconteceram em $33,5 \%$ dos casos.

A principal causa de óbito por acidentes na faixa etária de 1 a 4 anos foi por afogamentos e submersões acidentais, tanto para macrorregião Jequitinhonha $(45,2 \%)$ quanto para a macrorregião Centro $(24,7 \%)$. Os acidentes nesta faixa etária foram responsáveis por $17,5 \%$ dos óbitos no Vale do
Jequitinhonha, e por $1,4 \%$ dos óbitos na macrorregião Centro.

Crianças em geral estão mais vulneráveis à ocorrência de acidentes. No entanto, alguns fatores como a falta de informação, de infra-estrutura adequada, de espaços de lazer, de creches e escolas e de políticas públicas voltadas para a prevenção do acidente estão ligados ao aumento da exposição das crianças ao risco (FREITAS; RAMIRES, 2012). Melhor compreensão contextual dos fatores que predispõem as crianças ao risco de injúrias acidentais é importante para garantir medidas de controle mais eficazes (BALAN, LINGAM, 2012).

A incidência dos acidentes é de difícil estimação, pois, no âmbito da saúde pública, utilizam-se dados de eventos vitais em que são computadas apenas as causas externas que tiveram como desfecho o óbito. Somada a esta dificuldade, dados de alta qualidade relativos ao uso de serviços hospitalares são escassos e de difícil acesso. Assim, a falta geral de dados sobre os cuidados de saúde, em particular sobre o atendimento às crianças $\mathrm{e}$ adolescentes vítimas de acidentes, dificulta a elaboração de estratégias organizacionais e políticas conforme as necessidades regionais bem como inibe a alocação de recursos para esforços no sentido de prevenção ao acidente.

A distribuição epidemiológica dos acidentes que acarretam em morte difere do que é normalmente encontrado para acidentes relacionados à morbidade (WAKSMAN et al, 
2014). Este estudo, per si, não permite concluir acerca da incidência dos acidentes uma vez que foram avaliados apenas dados relacionados ao desfecho óbito.

Até o momento, pouquíssimas pesquisas foram publicadas avaliando as especificidades regionais do Vale do Jequitinhonha. Além disso, inexistem estudos descritivos que avaliem o perfil epidemiológico dos acidentes infanto-juvenis nesta região. Compará-la com a região central do estado significa ter um parâmetro do que representaria estar mais próximo de condições de saúde mais satisfatórias, por englobar municípios com maior infra-estrutura e profissionais de saúde mais especializados.

Este estudo permite duas suposições que podem ser tema de trabalhos futuros: (a) o vale do Jequitinhonha necessita de políticas voltadas à prevenção de acidentes por submersão, de forma que um levantamento epidemiológico específico sobre este tipo de acidente seria de grande relevância para direcionar estas políticas; (b) o Vale do Jequitinhonha necessita de políticas voltadas à melhor estruturação da rede de atenção à saúde, de forma que possa absorver as demandas dentro do seu próprio território de saúde, garantido resolubilidade e evitando óbitos infanto-juvenis.

A supervisão das crianças é um reconhecido fator determinante na prevenção da ocorrência de injúrias acidentais (WAKSMAN et al, 2014). Na busca de

prevenir o acontecimento de eventos acidentais com crianças e adolescentes, sugere-se que as estratégias saúde da família possam contribuir promovendo atividades informativas próximas à realidade social das comunidades. Neste contexto, a capacitação dos profissionais voltada para uma prática educativa teria papel fundamental na condução de um trabalho educativo com a população, que deve explorar o campo da informação e integrar a consideração de valores, de costumes, de modelos e de símbolos sociais existentes (BESEN et al, 2007).

Em relação às medidas de prevenção da mortalidade por estes eventos, ressalta-se a importância da criação e implantação de protocolos de atendimento às crianças $\mathrm{e}$ adolescentes vítimas de acidentes e o treinamento de profissionais da rede de urgência e emergência para atendimento deste perfil de pacientes (MARTINS; ANDRADE, 2008).

O presente trabalho apresenta limitações relacionadas à existência de subregistros, erros no preenchimento das declarações de óbitos, falhas da cobertura do sistema e perdas na transmissão dos dados do SIM/MS. Respeitadas as limitações de qualidade e abrangência dos dados, eles são de extrema relevância no contexto das análises das diferenças nos padrões de ocorrências dos acidentes infanto-juvenis. Este tipo de estudo pode contribuir no 
direcionamento de estratégias voltadas para grupos específicos conforme exposição aos fatores de riscos identificados.

\section{Conclusão}

O levantamento epidemiológico realizado aponta que no Vale do Jequitinhonha prevalecem óbitos infantojuvenis associados a fatores contextuais e culturais típicos do território estudado. Afogamento e submersão acidental foram as maiores causas de óbito por acidentes nesta região. Além disso, o estudo mostra que, mesmo nesta região onde o tráfego de veículos é consideravelmente menor se comparado à região central do estado, as consequiências dos acidentes de motocicletas constituem-se um dos grandes agravos à saúde da localidade. Convém salientar que o levantamento desta problemática pode orientar abordagens técnico-científicas para minimização da ocorrência de acidentes na faixa etária estudada.

\section{Referências}

BALAN B, LINGAM L. Unintentional injuries among children in resource poor settings: where do the fingers point? Archives of Disease in Childhood, United Kingdon, v.97, n.1, p.35-38, 2012.

\section{BASTOS YGL, ANDRADE SM, SOARES}

DA. Características dos acidentes de trânsito e das vítimas atendidas em serviço préhospitalar em cidade do Sul do Brasil, 1997/2000. Cadernos de Saúde Pública. Rio de Janeiro, v.21, n.3, p. 815-822, 2005.
BESEN CB, NETTO MS, DA ROS MA, SILVA FW, SILVA CG, PIRES MF. A Estratégia Saúde da Família como Objeto de Educação em Saúde. Saúde e Sociedade, São Paulo, v.16, n.1, p.57-68, 2007.

BLANK D. Prevenção e controle de injúrias físicas: saímos ou não do século 20? Jornal de Pediatria, Rio de Janeiro, v.78, n.2, p.8486, 2002.

BRASIL. Ministério da Saúde. Departamento de Análise de Situação de Saúde, Secretaria de Vigilância em Saúde, Ministério da Saúde. Saúde Brasil 2011: uma análise da situação de saúde e a vigilância da saúde da mulher. Brasília: Ministério da Saúde; 2012.

FREITAS JPP, RAMIRES JCL. Cities and chaos: road traffic and its reflexes on children health. Hygeia, Uberlândia. v.8, n.15, p.137145, 2012.

GALVÃO EL, BODEVAN EC, SANTOS DF. Análise da distribuição geográfica dos serviços de saúde no Vale do Jequitinhonha, Minas Gerais. Hygeia, Uberlândia, v.11, n.20, p.32-44, 2015.

GAWRYSZEWSKI VP, KOIZUMI MS, MELLO-JORGE MHP, As causas externas no Brasil no ano 2000: comparando a mortalidade e a morbidade. Cadernos de Saúde Pública, Rio de Janeiro, v.20, n.4, p.995-1003, 2004.

INSTITUTO DE PESQUISA ECONÔMICA APLICADA- IPEA. Atlas de vulnerabilidade social nos municípios brasileiros. Brasília: IPEA, 2015. [citado 2016 mar 19]; Disponível em:http://ivs.ipea.gov.br/ivs/data/rawData/pu blicacao_atlas_ivs.pdf

LIBERATTI CLB, ANDRADE SM, SOARES DA, MATSUO T. Uso de capacete por vítimas de acidentes de motocicleta em Londrina, sul do Brasil. Revista Panamericana de Salud Pública, Washington, v.13, n.1, p.33-38, 2003. 
MARTINS CBG, ANDRADE SM. Acidentes com corpo estranho em menores de 15 anos: análise epidemiológica dos atendimentos em pronto-socorro, internações e óbitos.

Cadernos de Saúde Pública, Rio de Janeiro, v.24, n.9, p.1983-1990, 2008.

MARTINS CBG. Acidentes na infância e adolescência: uma revisão bibliográfica. Revista Brasileira de Enfermagem, Brasília, v.59, n.3, p.344-348, 2006.

MINAS GERAIS. Secretaria de Estado de Saúde (SES-MG). Plano Diretor de

Regionalização: PDR 2011. [Internet]. [citado 2016 abr 11]. Disponível em:

http://www.saude.mg.gov.br/images/documen tos/Livro\%20Plano\%20Diretor\%20de\%20Re gionalizacao\%20-\%20ultima\%20versao.pdf

MINAYO MCS. Seis características das mortes violentas no Brasil. Revista Brasileira de Estudos de População, Rio de Janeiro, v.26, n.1, p.135-140, 2009.

BRASIL. Ministério da Saúde. DATASUS. Sistema de Informação sobre Mortalidade 1998-2013. [Internet]. [citado 2016 mai 10]. Disponível em: http://www.datasus.gov.br.

PAES EN, GASPAR VLV. Unintentional injuries in the home environment: home safety. Jornal de Pediatria. Rio de Janeiro, v.81, n.5, [Supl], p.S146:S154, 2005.

SCHRAMM JMA, OLIVEIRA AF, LEITE IC, VALENTE JG, GADELHA AMJ, PORTELA MC, CAMPOS MR. Transição epidemiológica e o estudo de carga de doença no Brasil. Ciência e Saúde Coletiva, Rio de Janeiro, v.9, n.4, p.897-908, 2004.

WAKSMAN RD, CARRERA RM, SANTOS E, ABRAMOVICI, SCHVARTSMAN C. Morbidity due trauma in children of the community of Paraisopolis, São Paulo, Brazil. Einstein, São Paulo, v.12, n.1, p.1-5, 2014.

ORGANIZAÇÃO MUNDIAL DE SAÚDE.

World report on child injury prevention.

Geneva: World Health Organization; 2008. 